\title{
Conversion of Neutron Stars to Strange Stars as a Possible Origin of $\gamma$-Ray Bursts
}

\author{
K. S. Cheng ${ }^{1}$ and Z. G. Dai ${ }^{2}$ \\ ${ }^{1}$ Department of Physics, University of Hong Kong, Hong Kong \\ ${ }^{2}$ Department of Astronomy, Nanjing University, Nanjing 210093, China
}

(Received 21 November 1995)

\begin{abstract}
We propose that some neutron stars in low-mass x-ray binaries can accrete sufficient mass to undergo a phase transition to become strange stars. The energy released per conversion event satisfies the requirements of cosmological $\gamma$-ray bursts, and the Lorentz factor of the resultant expanding fireball may exceed $5 \times 10^{3}$ because the strange star has very low baryon contamination. The model burst rate is consistent with observations. [S0031-9007(96)00892-7]
\end{abstract}

PACS numbers: 98.70.Rz, 12.38.Mh, 26.60.+c, 97.60.Jd

The recent observational results from the BATSE detector on the Compton Gamma-Ray Observatory [1] strongly suggest that the sources of weak $\gamma$-ray bursts are at cosmological distances [2]. Prior to the BATSE observations, several authors [3] proposed that $\gamma$-ray bursts arise at cosmological distances in the merger of binaries consisting of either two neutron stars or a neutron star and black hole, while other authors [4] suggested that $\gamma$-ray bursts result from phase transition of normal nuclear matter to matter with pion condensation in neutron stars. Now the cosmological scenarios also include the collapse of a white dwarf to a neutron star with an extremely strong magnetic field and the formation of a transient accretion disk around a black hole resulting from a failed type $\mathrm{Ib}$ supernova [5]. In this Letter we argue that the conversion of neutron stars to strange stars is another possible origin of $\gamma$-ray bursts. Such converting stars may be neutron stars in the binaries with low-mass companions.

When nuclear matter is squeezed to a sufficiently high density, it turns into uniform two-flavor quark ( $u$ and $d$ ) matter. But the quark matter is unstable, and subsequently converts to three-flavor (strange) quark matter, due to the fact that strange matter may be more stable than nuclear matter [6]. The properties of strange stars have been studied [7]; however, their existence is doubtful for several reasons.

First, it has not yet been confirmed that strangequark matter is a stable form of matter. The results from ultrarelativistic heavy-ion collisions are expected to provide solid proof [8], and, in fact, recent experimental results do suggest some evidence of the existence of strange-quark condensation [9]. However, the conclusion is still controversial, and further theoretical studies in the properties of strange-quark matter, especially the exact conditions for the phase transition and experiments in the higher energy range, are necessary [10].

Second, other objections against the existence of strange stars result from astrophysical arguments. It has been argued that the disruption of a single strange star can contaminate the entire galaxy and essentially all "neutron" stars are strange stars [11]. This conflicts with the relaxation behavior of pulsar glitches, which is well described by the neutron-superfluid vortex creep theory [12], and current strange-star models scarcely explain the observed pulsar glitches. Furthermore, if $\gamma$-ray bursts are the consequence of the merger of two compact stars, even a single merger event involves a strange star, and all pulsars are then strange stars [13]. However, we want to comment that these arguments do not necessarily disprove the existence of strange stars for the following reasons. (i) In order to disrupt a strange star, its companion must also be a very compact object, e.g., a neutron star or a black hole but not a white dwarf because the tidal force must be strong enough to disrupt the strange star. According to the standard evolution model of millisecond pulsars, which is expected to be the progenitor of strange stars in our model, its companion is a low-mass white dwarf. The subsequent evaporation process can further reduce the mass of the white dwarf and may eventually totally remove this companion star [14]. At least in this model, disruption will not take place. (ii) Even in the case of neutron-star-neutron-star merger or neutron-starblack-hole merger, how much material will be shed is still an open question [15]. (iii) If the core of a strange star consists of quantized fluxoids and vortex lines, then the relaxation of a strange-star glitch will be very similar to that of ordinary neutron stars [16].

Third, the conversion of a neutron star to a strange star requires the formation of a strange-matter seed in the star, which is produced through the deconfinement of neutron matter at a density, sufficiently larger than the central density of the $1.4 M_{\odot}$ star with a rather stiff equation of state [17]. In view of these uncertainties, we should only regard strange stars as strong possible stellar objects.

It is thought that the density for deconfinement of neutron-star matter with a moderately stiff (or stiff) equation of state to two-flavor quark matter is near $8 \rho_{0}$ (where $\rho_{0}$ is the nuclear density). For a soft equation of state, the deconfinement density is lower. Here we assume that the equations of state in neutron stars are moderately stiff or stiff. This is because soft equations of state at high densities are ruled out by the postglitch 
recovery in four pulsars [18]. More detailed analyses of the postglitch curves of the Crab and Vela pulsars also draw similar conclusions [19]. In addition, the soft equation of state, as in kaon condensation, seems not to occur in stable neutron stars [20]. The neutron stars with $1.4 M_{\odot}$, based on the modern equations of state [21] named UV14+UVII, AV14+UVII, and UV14+TNI, must accrete matter of $\sim 0.6 M_{\odot}, 0.5 M_{\odot}$, and $0.4 M_{\odot}$ in order that their central densities reach the deconfinement density. Once this condition is satisfied, strange-matter seeds are formed in the interiors of the stars.

After a strange-matter seed is formed, the strange matter will begin to swallow the neutron matter in the surroundings. While it has been proposed [22] that the combustion corresponds to the slow mode, subsequent work [23] shows that this mode appears to be hydrodynamically unstable. Thus the conversion of neutron matter should proceed in a detonation mode. The total kinetic reaction of the detonation mode has two stages: the formation of two-flavor quark matter and the weak decays that form strange matter. Since the second process enhances the thermal energy at the expense of the chemical energy of two-flavor quark matter [24], the temperature in the stellar interior will increase to more than $10 \mathrm{MeV}$. In addition, the time scale [23] for the conversion of a neutron star to a strange star is smaller than $1 \mathrm{~s}$.

The resulting strange star [25] has a thin crust with mass $\sim 2 \times 10^{-5} M_{\odot}$ and thickness $\sim 150 \mathrm{~m}$, but because the internal temperature is so high $\left(\sim 10^{11} \mathrm{~K}\right)$, the nuclei in this crust may decompose into nucleons. Approximating strange matter by a free Fermi gas, we obtain the total thermal energy of the star, $E_{\mathrm{th}} \sim 5 \times$ $10^{51} \operatorname{ergs}\left(\rho / \rho_{0}\right)^{2 / 3} R_{6}^{3} T_{11}^{2}$, where $\rho$ is the average mass density, $R_{6}$ the stellar radius in units of $10^{6} \mathrm{~cm}$, and $T_{11}$ the temperature in units of $10^{11} \mathrm{~K}$. Adopting $\rho=8 \rho_{0}$, $R_{6}=1$, and $T_{11}=1.5$, we have $E_{\mathrm{th}} \sim 5 \times 10^{52}$ ergs.

The star will cool by the emission of neutrinos and antineutrinos, and because of the huge neutrino number density, the neutrino pair annihilation process $\nu \bar{\nu} \rightarrow e^{+} e^{-}$ operates in the region close to the strange-star surface. The total energy [26] deposited due to this process is $E_{1} \sim 2 \times 10^{48} \operatorname{ergs}\left(T_{0} / 10^{11} \mathrm{~K}\right)^{4} \sim 10^{49} \operatorname{ergs}\left(\right.$ where $T_{0}$ is the initial temperature) and the time scale for deposition is of the order of $1 \mathrm{~s}$. On the other hand, the processes for $n+\nu_{e} \rightarrow p+e^{-}$and $p+\bar{\nu}_{e} \rightarrow$ $n+e^{+}$play an important role in the energy deposition, and the integrated neutrino optical depth [27] due to these processes is $\tau \sim 4.5 \times 10^{-2} \rho_{11}^{4 / 3} T_{11}^{2}$ (where $\rho_{11}$ is the crust density in units of $10^{11} \mathrm{~g} \mathrm{~cm}^{-3}$ ). So the deposition energy is estimated by $E_{2} \sim E_{\mathrm{th}}\left(1-e^{-\tau}\right) \sim$ $2 \times 10^{52}$ ergs. Here we have used the neutron-drip density $\left(\rho_{11} \sim 4.3\right)$, and have assumed that the thermal energy of the star is wholly lost in neutrinos. The process, $\gamma \gamma \leftrightarrow e^{+} e^{-}$, inevitably leads to the creation of a fireball. However, the fireball must be contaminated by the baryons in the thin crust of the strange star. If we define $\eta=E_{0} / M_{0} c^{2}$, where $E_{0}=E_{1}+E_{2}$ is the initial radiation energy produced $\left(e^{+} e^{-}, \gamma\right)$ and $M_{0}$ is the conserved rest mass of baryons with which the fireball is loaded, then, since the amount of the baryons contaminating the fireball cannot exceed the mass of the thin crust, we have $\eta \geq 5 \times 10^{3}$ and the fireball will expand outward. The expanding shell (having a relativistic factor $\Gamma \sim \eta$ ) interacts with the surrounding interstellar medium and its kinetic energy is finally radiated through nonthermal processes in shocks [28].

What mechanism results in the conversion of neutron stars? Here we propose that accretion in binaries with low-mass companions can lead to the conversion. It has been shown [29] that the amount of matter accreted $(\Delta M)$ by the 18 radio pulsars in these binary systems exceeds $0.5 M_{\odot}$. If this is true, some of the millisecond pulsars should have masses over $2 M_{\odot}$, and they may be strange stars. By assuming that the number of galaxies at cosmological distances is $\mathcal{N}$, and the number of neutron stars in low-mass $\mathrm{x}$-ray binaries which will convert into strange stars in the characteristic accretion time scale $(\sim \Delta M / \dot{M})$ is $N_{B}$, we have the burst rate

$$
\begin{aligned}
\mathcal{R} & \sim \frac{\mathcal{N} N_{\mathrm{B}}}{\Delta M / \dot{M}} \\
& \sim 10 \text { day }^{-1}\left(\frac{\mathcal{N}}{10^{10}}\right)\left(\frac{N_{B}}{10}\right)\left(\frac{\Delta M}{0.5 M_{\odot}}\right)^{-1}\left(\frac{\dot{M}}{\dot{M}_{\text {Edd }}}\right),
\end{aligned}
$$

where $\dot{M}$ is the accretion rate and $\dot{M}_{\text {Edd }}$ is the Eddington accretion rate. In order to determine the conversion rate, we need to estimate the value of $N_{B}$.

The direct criterion for the conversion of neutron stars to strange stars is that the total mass of the accreting neutron stars should exceed $2 M_{\odot}$. Observationally, only the mass function can be determined precisely but not the mass of the individual star. The one exception is the star in the Hulse-Taylor binary system, because the high precision of pulsar measurements combined with the relatively high orbital velocity of the system has allowed measurements of the general relativistic periastron advance and second order Doppler shift [30]. However, there are indirect ways to estimate the conversion rate. First, if a good fraction of neutron stars in luminous low-mass X-ray binaries (LMXBs) is converted into strange stars, then $N_{B}$ is close to the current observed number of LMXBs $(\sim 10)$ [31]. Second, We expect that possible strange-star candidates are very weak-field millisecond pulsars which have a lifetime near the Hubble time. If the radio beams of the millisecond pulsars are fan beams [32], there are about $10^{3}$ to $10^{4}$ weak-field millisecond pulsars in our galaxy as estimated by scaling from the present observed values [33]. Thus, the conversion rate is about $(3-30) \times 10^{-10}$ per day per galaxy. This conversion rate seems consistent with the $\gamma$-ray burst rate. Here we want to add two remarks. (1) The companion of the strange star is a white 
dwarf in the accretion induced spun-up scenario, and the pulsar wind may evaporate this white dwarf eventually [14]. (2) The fact that the surface magnetic fields of millisecond pulsars seem independent of the amount of accretion material [29] and that there is a gap in the magnetic field distribution of pulsars $[31,34]$ are suggested as evidence of the phase transition from neutron stars to strange stars, because these two kinds of compact objects have different minimum magnetic fields which can be supported by the stellar crusts [35].

It is well known that the merging of two neutron stars has been proposed as a possible origin for cosmological $\gamma$-ray bursts [3]. Our converting model differs from the merging model as follows. First, the merging should produce observable gravitational waves [36], but there are no gravitational radiations in our model if the conversion is spherically symmetric. Future observation of gravitational waves may distinguish between the converting and merging processes. Second, the formation rate of compact binaries is quite uncertain, but a current estimation [37] lies in the range $10^{-5}-10^{-4} \mathrm{yr}^{-1}$ per galaxy. Thus, the merging rate seems to be much larger than the observed burst rate. In our scenario, the estimated rate is consistent with the burst rate. Third, because the strange star just formed during the conversion has a very thin crust, the resultant fireball is contaminated by a small amount of baryons $\leq 10^{-5} M_{\odot}$, but in the merging model the number of baryons loaded with the fireball is unlikely to be small [27]. Therefore, the evolution of the fireball in the conversion model is somewhat different from that in the merging model.

We would like to thank M. A. Alpar, S. Bludman, H. F. Chau, M. C. Chu, J. Fry, S. Ichimaru, W. Kluzniak, F. Lamb, T. Piran, M. A. Ruderman, N. Shibazaki, W. M. Suen, J. W. Truran, and E. P. J. van den Heuvel for many enlightening discussions, and T. Boyce for a critical reading of our paper.

[1] G. J. Fishman and C.A. Meegan, Annu. Rev. Astron. Astrophys. 33, 325 (1995).

[2] S. Mao and B. Paczyński, Astrophys. J. Lett. 388, L45 (1992); T. Piran, Astrophys. J. Lett. 389, L45 (1992); C. D. Dermer, Phys. Rev. Lett. 68, 1799 (1992).

[3] B. Paczyński, Astrophys. J. Lett. 308, L43 (1986); D. Eichler, M. Livio, T. Piran, and D. N. Schramm, Nature (London) 340, 126 (1989); R. Narayan, T. Piran, and A. Shemi, Astrophys. J. Lett. 379, L17 (1991); B. Paczyński, Acta Astron. 41, 257 (1991).

[4] P. Haensel and B. Proszynski, Astrophys. J. 258, 306 (1982); P. Haensel et al., Astron. Astrophys. 240, 78 (1990); T. Muto et al., Prog. Theor. Phys. Suppl. 112, 221 (1993).

[5] V. V. Usov, Nature (London) 357, 472 (1992); R. Duncan and C. Thompson, Astrophys. J. Lett. 392, L9 (1992); S. E. Woosley, Astrophys. J. 405, 273 (1993).
[6] A. R. Bodmer, Phys. Rev. 4, 1601 (1971); H. Tarazawa, University of Tokyo, Report No. 338 (to be published); E. Witten, Phys. Rev. D 30, 272 (1984).

[7] C. Alcock, E. Farhi, and A. Olinto, Astrophys. J. 310, 261 (1986); P. Haensel, J. L. Zdunik, and R. Shaeffer, R. Astron. Astrophys. 160, 121 (1986).

[8] J. Rafelski, Phys. Rep. 88, 331 (1982).

[9] O. Hansen, in The Nuclear Equation of State, edited by W. Greiner and H. Stocker, NATO ASI Series 216B (Plenum, New York, 1990), p. 97; M. Gazdzicki, in The Nuclear Equation of State, edited by W. Greiner and H. Stocker, NATO ASI Series 216B (Plenum, New York, 1990), p. 103; V. Topor Pop et al., Phys. Rev. C 52, 1618 (1995).

[10] For a general review of recent developments of theoretical studies, experimental results, and future experimental plans in the role of strange-quark matter in ultrarelativistic heavy ion collisions, cf. J. Sollfrank and U. Heinz, in Quark-Gluon Plasma 2, edited by R.C. Hwa (World Scientific, Singapore, 1996).

[11] R. R. Caldwell and J. R. Friedman, Phys. Lett. B 264, 143 (1991).

[12] D. Pines and M. A. Alpar, Nature (London) 316, 27 (1985).

[13] W. Kluźniak, Astron. Astrophys. 286, L17 (1994).

[14] M. A. Alpar, A. Cheng, M. A. Ruderman, and J. Shaham, Nature (London) 300, 728 (1982); E. S. Phinney, C. R. Evans, R. D. Blandford, and S. R. Kulkarni, Nature (London) 333, 832 (1988).

[15] W. M. Suen (private communication).

[16] H.F. Chau, K. S. Cheng, and K. Y. Ding, Astrophys. J. 399, 213 (1992); H. F. Chau, Princeton University, Report No. IASSNS-HEP-96/16 (to be published).

[17] B. D. Serot and H. Uechi, Ann. Phys. (N.Y.) 179, 272 (1987); G. Baym, in Neutron Stars: Theory and Observation, edited by J. Ventura and D. Pines (Kluwer, Dordrecht, 1991).

[18] B. Link, R.I. Epstein, and K. A. Van Riper, Nature (London) 359, 616 (1992).

[19] M. A. Alpar, H.F. Chau, K.S. Cheng, and D. Pines, Astrophys. J. 409, 345 (1993); M. A. Alpar, H. F. Chau, K. S. Cheng, and D. Pines, Astrophys. J. Lett. 427, L29 (1994).

[20] V. R. Pandharipande, C. J. Pethick, and V. Thorsson, Phys. Rev. Lett. 75, 4567 (1995).

[21] R. B. Wiringa, V. Fiks, and A. Fabrocini, Phys. Rev. C 38, 1010 (1988).

[22] A. Olinto, Phys. Lett. B 192, 71 (1987).

[23] J. E. Horvath and O. G. Benvenuto, Phys. Lett. B 213, 516 (1988).

[24] Z. G. Dai, Q. H. Peng, and T. Lu, Astrophys. J. 440, 815 (1995).

[25] N. K. Glendenning and F. Weber, Astrophys. J. 400, 647 (1992).

[26] P. Haensel, B. Paczyński, and P. Amsterdamski, Astrophys. J. 375, 209 (1991).

[27] P. Mészáros and M. Rees, Astrophys. J. 397, 570 (1992).

[28] P. Mészáros and M. Rees, Astrophys. J. 405, 278 (1993).

[29] E.P. J. van den Heuvel and O. Bitzaraki, Astron. Astrophys. 297, L41 (1995). 
[30] J. H. Taylor and J. M. Weisberg, Astrophys. J. 253, 908 (1982).

[31] F. Camilo (to be published).

[32] K. Chen and M. A. Ruderman, Astrophys. J. 408, 179 (1993).

[33] J. H. Taylor, R. N. Manchester, and A. G. Lyne, Astrophys. J. Suppl. 88, 529 (1993).
[34] F. Camilo, S. E. Thorsett, and S. R. Kulkarni, Astrophys. J. Lett. 421, L15 (1994).

[35] K. S. Cheng and Z. G. Dai (to be published).

[36] C. S. Kochanek and T. Piran, Astrophys. J. Lett. 417, L17 (1993).

[37] A. V. Tutukov and L. R. Yungelson, Mon. Not. R. Astron. Soc. 260, 675 (1993). 